\title{
An Adaptive and Cost-Effective Data Transmission Framework Using MMS Technology for Client-Server Application
}

\author{
http://dx.doi.org/10.3991/ijim.v6i1.1760 \\ A. Setyono ${ }^{1}$, M. J. Alam² and C. Eswaran ${ }^{3}$ \\ 1, 2, 3 Multimedia University, Cyberjaya, Malaysia \\ ${ }^{1}$ Dian Nuswantoro University, Semarang, Indonesia
}

\begin{abstract}
Limitation of message size of the MMS technology is one of the important factors which make this technology less attractive. This limitation restricts the system developers in developing new mobile applications using MMS technology. This research attempts to improve the capacity of the maximum message size by implementing compression, splitting and cropping techniques for MMS application on the mobile phone devices. This paper presents the architecture to enhance the multimedia capabilities of the MMS technology and also the implementation details for developing client-server system applications. The modified MMS technology can be used to develop practical client-server applications.
\end{abstract}

Index Terms-Multimedia message, Message size, MMS, client-server application

\section{INTRODUCTION}

Multimedia messaging service (MMS) provides the user a complete knowledge about multimedia and has the potential to significantly increase the mobile communication usage [1]. MMS is considered as the best and proven messaging technology and it has surpassed email and SMS [2]. MMS experienced dramatic growth as for example in the USA, more than 10.3 billion MMS messages were sent in the first half of 2009 and experienced a growth of 4.7 billion from the same period of the previous year [3]. The initial standard for MMS size was up to a maximum of $30 \mathrm{~KB}$. The standard was raised to $100 \mathrm{~KB}$ and the 3G standard is $300 \mathrm{~KB}$ [3], [4]. Currently, MMS supports an average message size of $600 \mathrm{~KB}$ with maximum message size limited only by the handset's and MMS Center (MMSC) capabilities. The standard is not used widely yet, because it must be supported by the availability of sophisticated mobile phone and MMSC capability [5].

Due to the authorization problem, we cannot change the message size that is received and forwarded to the recipient. We use the MMS technology standard that has been provided by the operator. We develop a data transmission method with improved MMS technology for client-server application. We attempt to make MMS technology more adaptive and cost-effective to overcome the limitations in the maximum size for developing client-server applications.

The main goals of this research are: (i) to explore data transmission for large multimedia file using MMS technology (ii) to develop data transmission by implementing the proposed techniques (iii) to design an adaptive and cost-effective client-server application and (iv) to implement the techniques for developing client-server application. The developed system will be used to implement the client-server application, with MMS application on the client side and Internet applications on the server side. Internet applications on the server side can be accessed by user with MMS application in mobile environment. This research is expected to provide a valuable contribution to the readers and researchers for developing mobile system applications using MMS technology. The following are the important contributions of this paper: (i) it describes about the mobile messaging system (ii) it develops transmission method for large multimedia file size (iii) it presents the design of an adaptive and cost-effective for client-server applications and (iv) it also implements and discusses about an adaptive and cost-effective transmission method for mobile system application.

The rest of the paper is organized as follows: Section 2 discusses data transmission method. The proposed design of client-server application and discussion is presented in Section 3. Implementation and simulation are presented in Section 4. Section 5 discusses the evaluation and analysis and finally conclusion and future work are given in Section 6.

\section{DATA TRANSMISSION TECHNIQUES}

MMS technology is the developed version of the SMS technology [2]. SMS has experienced tremendous success that cannot be exceeded by MMS [4]. In the early stages, many people expected that the MMS could replace SMS, as it has the advantage of sending multimedia data [6]. However, the growth of MMS technology was restricted because of reasons such as high-cost of sending multimedia message, high-prices of mobile devices and message size limitations [7]. In multimedia data transmission, MMS technology uses 2.5G or 3G network with speeds up to $384 \mathrm{Kbps}$ [8]. In this work, we make some improvements on the MMS application by implementing a few techniques before data transmission to the recipient user.

The MMS server is more known as MMS center (MMSC) that is the center of the MMS system. MMSC performs a lot of functions, such as store MMS messages, forward MMS messages to external networks, send MMS messages as email using the SMTP protocol over the Internet, send MMS messages via the SMS for enabling users of non-MMS phones to receive MMS messages and 


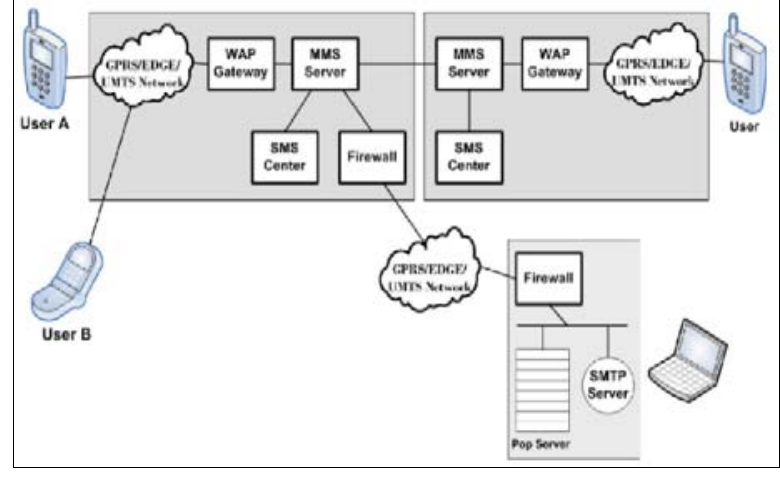

Figure 1. MMS architecture for data transmission

view the text as SMS messages and the whole message via Web or WAP and perform content adaptation [3]. The client-server applications have a client program which is running on one end of the system that requests and receives a service from server program running on another end system. This will be different if the client-server programs running on the computer, it is called a distributed application [9]. Figure 1 shows the MMS architecture for data transmission.

On the client side, we develop MMS application by implementing some techniques, such as compression, splitting, masking and cropping to get an adaptive and costeffective in message delivery of the large multimedia file. Then, on the server side, we develop Internet application to use the data which is sent by the user to the mobile phone recipient which serves as the gateway between client and server. We implement merging and decompression techniques when downloading data from gateway to store into the database server.

Currently, MMS technology still uses 2.5G or 3G mobile network to transmit multimedia message, where MMS does not use higher technology, such as 3.5G or HSDPA. The development of MMS technology should be supported by the ability of MMSC that is provided by the operator and mobile phone features such as camera, voice recording and video. In future, we believe that MMS technology will be able to send larger data than now, and then implementation techniques on the MMS application will give a few contributions to advancement of MMS technology. The use of MMS technology is easier (like sending SMS) compared to making Internet connection on mobile phone to access Internet applications. Besides that, we can make the system more secured because the user sends the data without opening the system on the Internet to upload multimedia data directly. The developed system will be an integrated system. Table 1 shows the mobile network capability for MMS technology.

Currently, MMS technology performs resizing process to send larger multimedia message but the maximum size is determined by MMSC and MMS features on the mobile phone. As this process reduces the quality of image and file size, the user is not able to send larger multimedia data without degrading the quality. The quality of handsets is improving rapidly with high quality of camera and screen resolution which allow users to send large contents of multimedia message more easily. Good quality of data is essential for consumers, but it increases the possibility that the message will become large. Operator increases the message size and ensures that its MMSC has high capacity and can handle the growth of messages efficiently.
TABLE I.

MOBILE NETWORK CAPABILITY FOR MMS TECHNOLOGY

\begin{tabular}{|l|r|r|}
\hline Technology & Data Rate & \multicolumn{1}{|c|}{ Frequency } \\
\hline Mobile Technology & & \\
GSM & $9.6 \mathrm{kbps}$ & $900 / 1800 / 1900 \mathrm{MHz}$ \\
GPRS & $171.2 \mathrm{kbps}$ & $900 / 1800 / 1900 \mathrm{MHz}$ \\
EDGE & $384 \mathrm{kbps}$ & $900 / 1800 / 1900 \mathrm{MHz}$ \\
3G / UMTS & $2 \mathrm{Mbps}$ & $1885-2200 \mathrm{MHz}$ \\
HSPDA & $14.4 \mathrm{Mbps}$ & $850 / 900 / 2100 \mathrm{MHz}$ \\
HSUPA & $5.76 \mathrm{Mbps}$ & $850 / 900 / 2100 \mathrm{MHz}$ \\
LTE & $100 \mathrm{Mbps}$ & $900 / 1700 / 1800 / / 2100 \mathrm{Mhz}$ \\
WiMAX & $1 \mathrm{Gbps}$ & $2 \mathrm{GHz}$ to $11 \mathrm{GHz}$ \\
\hline
\end{tabular}

In this research, we use data transmission method of the existing MMS protocol. We use mobile communications network that is provided by the service provider. We perform the development of MMS technology within the creation of MMS message by implementing some techniques in order to send large file size where the existing MMS technology cannot do it. This method is expected to be used in large multimedia message delivery

The developed system is a client server application. As we know that a mobile phone has many limitations compared to a personal computer (PC) or a notebook. Building the applications on mobile phone is rather complex considering its limitations. In developing the techniques to improve the ability of the MMS technology in sending the message, there are several factors that should be considered, such as resizing, converting, compressing, splitting, masking and cropping. These techniques can be used to overcome the problem of size limitation for data transmission. These techniques have advantages as well as limitations depending on the type of application. These techniques are briefly described below:

\section{A. Resizing}

This technique is widely used in the current MMS applications. Message that has a size in excess of the permitted capacity will be resized and as a result the quality will be reduced [10].

\section{B. Converting}

This technique is normally used in the MMS center to perform content adaptation process. For example, an audio file format allowed by the MMS center is AMR format. If we want to send an MP3 file, the MMS center must convert MP3 files into AMR before forwarding it to the recipient [11].

\section{Compressing}

Compression techniques can be used to overcome the problem of MM delivery of larger size files. By using the compression technique, we can reduce the file size, thus simplify the delivery process. This technique can also restore the quality as the original file is recovered using decompression techniques [12].

\section{Splitting}

This technique is used to split files, and then send these files one by one. The files are sent to the mobile phone recipient and then merged into a single file. This is similar to the technique used for sending an SMS [14].

\section{E. Masking}

Masking technique is used for masking a file. This technique is used to add a header for each split-file which can be received by MMSC. The masking technique can 
also be used to send the non-standardized file on MMS protocol by adding a header for each split-file message using the allowed image format, such as JPEG, PNG or GIF [14].

\section{F. Cropping}

Cropping is the process of removing portions of an image to focus or strengthen the composition. It means cutting part of object on image area to separate the objects from each other. This technique will reduce the image and file size [15].

In this research, we implement compression, splitting, masking and cropping techniques to develop MMS application. The compression technique is used to reduce the file size which is followed by a splitting process. The system splits the data if the compressed data file is still larger than the permitted MMS capacity. Masking technique is used to send audio or video files. The cropping technique is used to send a selected area of file (image) in a single message. The implementation of these techniques will lead to an adaptive and cost-effective client server application. We study and explore these techniques to improve the MMS technology.

\section{DESIGN OF ClIENT-SERVER APPLICATION}

\section{A. Design of Client Application}

We develop client-server application using Java programming language with its advantages and disadvantages. The system design on the client side is described through architecture design, mapping techniques and algorithms. MMS application is implemented by considering safety, efficiency, privacy, integrity and authentication. In terms of security, the message is encoded and split form, making it difficult for leaking of the information content in the message. Efficiency means that the user only pay the standard MMS tariff determined operator. The problem of privacy is also guaranteed because the user can not access the server directly, only the administrator can process data at the server. Integrity emphasizes that the information should not be altered without permission from the owner of the information, where the clientserver application is already integrated and automated. If message delivery is not up to the server, then the server will send notification to the sender automatically thus making the system robust. Figure 2 shows the overview of the architecture on the client side.

There are two main parts of the development in the MMS application, namely, delivery of large image files, and large audio and video files. Figure 3 shows the various techniques that are used to develop the MMS application on the client side.

Then, we also discuss the design of an algorithm for sending large multimedia messages. We expect that the outcome of this design can provide an adaptive and costeffective application. The MMS application is developed to send multimedia files of sizes which are larger than the allowed file sizes especially for image, audio and video data. They commonly have big file sizes so that we need to develop some techniques to transmit them from client to server side. We implement compression, splitting, cropping and masking techniques to enhance the MMS technology for transmitting multimedia files. We can use lossy or lossless compression, splitting based on pixel and bytes for image files and masking using image file for non-standard files that is not allowed to be sent using MMS technology. Figure 4 shows the step-by-step process of sending data from the sender to the recipient.

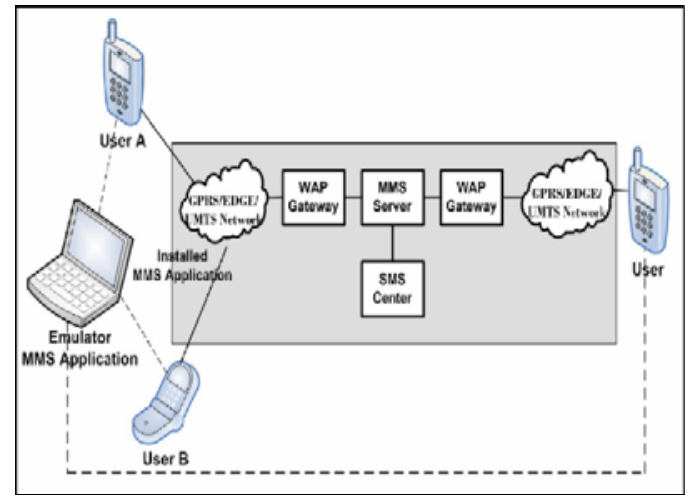

Figure 2. Overview of architecture on the client side

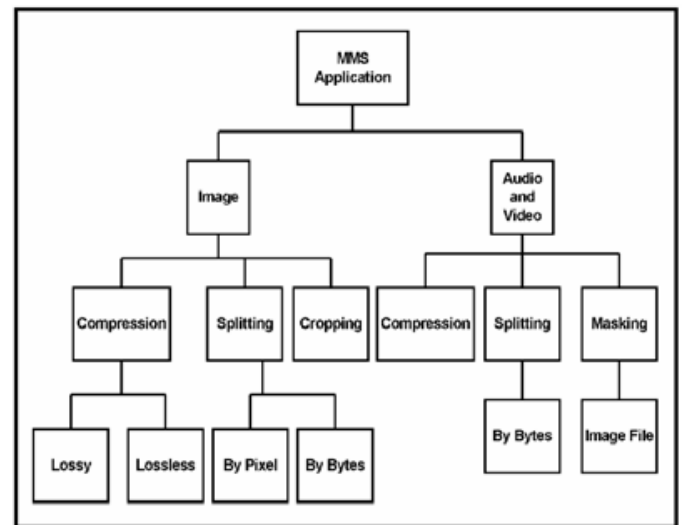

Figure 3. Techniques used to develop MMS application

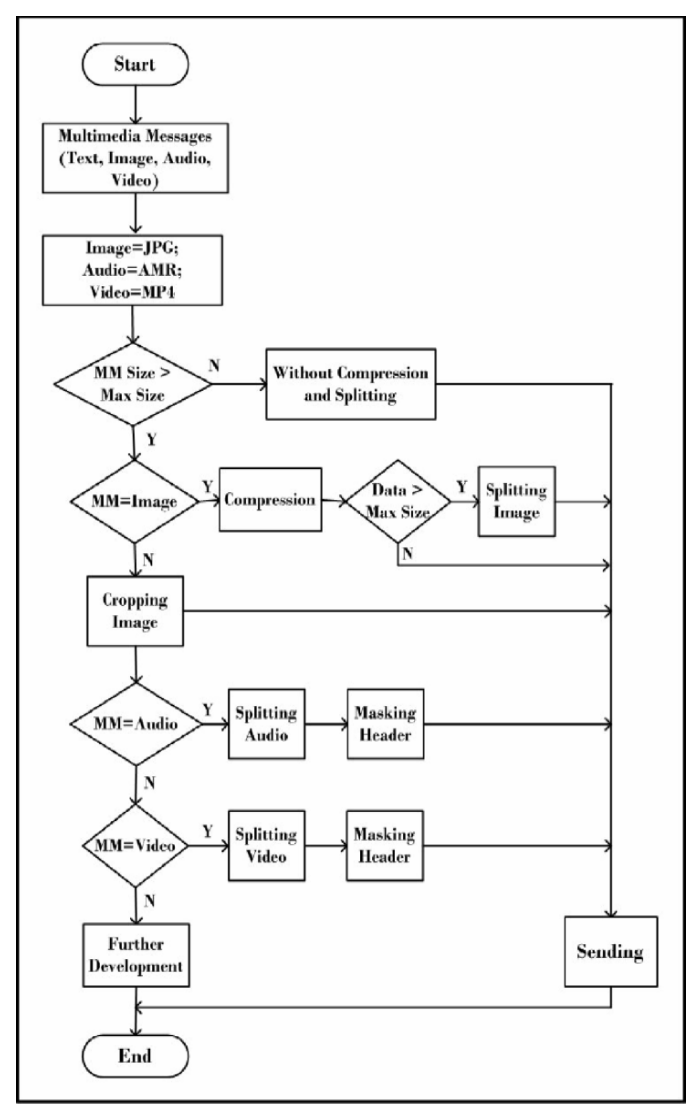

Figure 4. Flowchart for sending large multimedia file size 
MMS technology can be developed by implementing the compressing and splitting techniques. The implemented results of these techniques will lead to a MMS framework that is more efficient (cost-effective) and also adaptive for various sizes and types of files. Figure 5 shows the development of MMS technology to send large image file size.

The other technique that is used to improve the MMS technology is cropping technique. Figure 6 describes the algorithm in developing MMS technology using cropping technique.

The cropping will reduce the file size thus making the transmission and storage problems easier. The data quality is preserved because the collected data is uncompressed and only resized. To obtain better results, the cropping technique will be combined with compression. The unselected area of the image is also transmitted as this infor mation may also be needed in some cases. The unselected area can be compressed with high compression ratios to

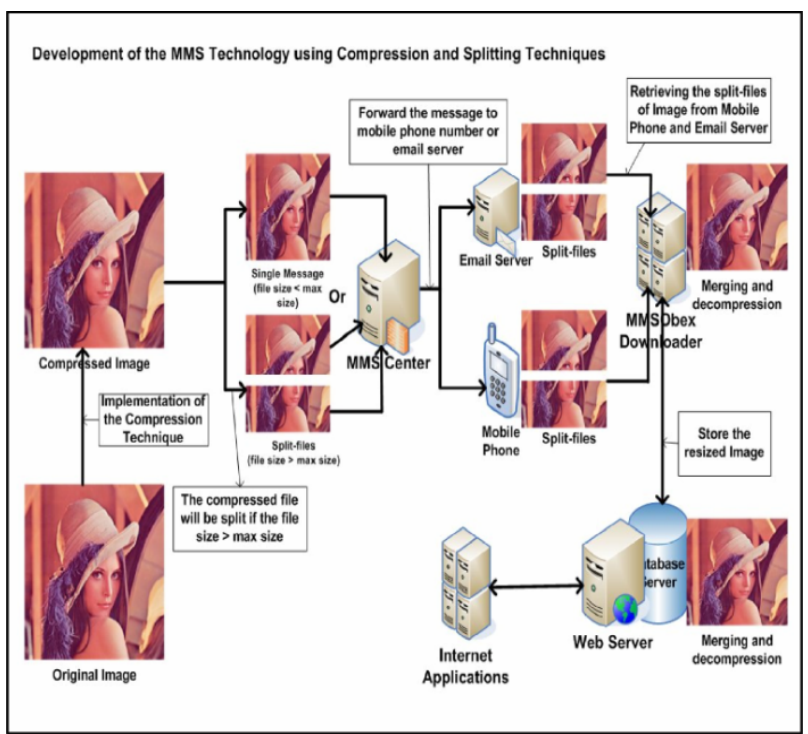

Figure 5. Development of MMS technology to send large image size

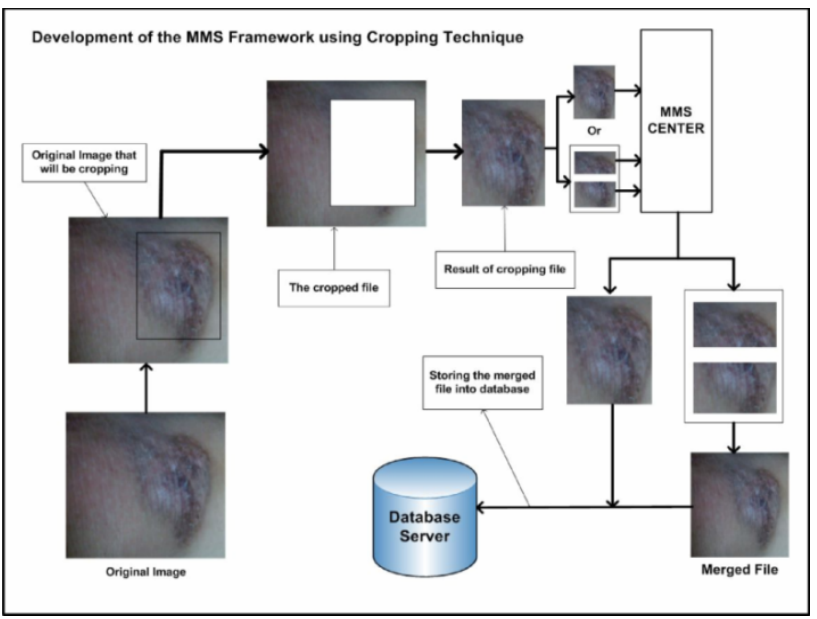

Figure 6. Development of the MMS using cropping technique reduce the file size, so that it can be transmitted together with the cropped file. Figure 7 describes the implementation of the MMS technology using compression and cropping techniques.

\section{B. Design of Server Application}

On the server side, we design an application for downloading, merging and decompressing data before it is stored into the database server. This framework can be used for a variety of Internet applications as client-server applications. Figure 8 shows the overview of the network architecture on the server side.

MMS data which is sent by the sender will be downloaded to move it to the server computer. Connection between mobile phone and the server computer must occur first. We use a data cable and Object Exchange (OBEX) downloader application. Figure 9 describes the algorithm for downloading data.

At the time of downloading the data, merging and decompression processes are performed. The development of server side application is not very complex as the client side. This is because the server computer can be easily customized to the application requirements which are not constrained by issues of memory and processing power.

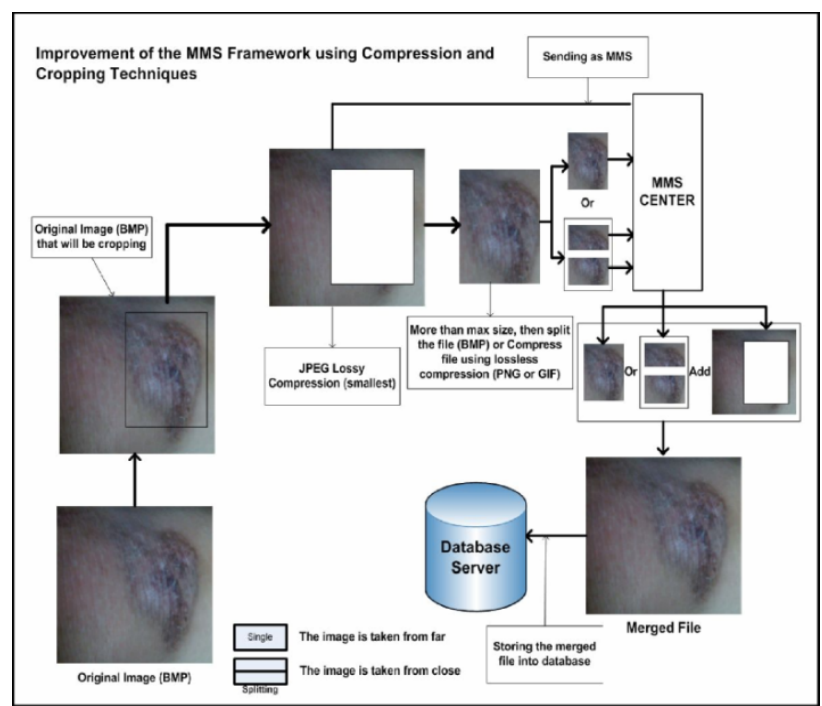

Figure 7. Improvement of the MMS using cropping technique

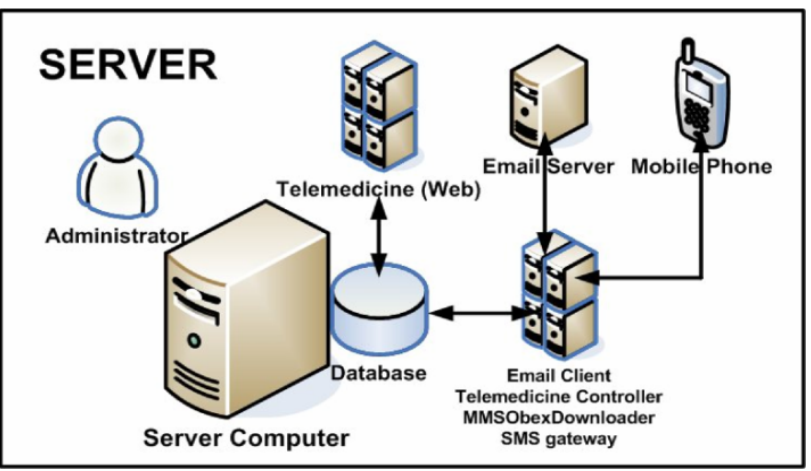

Figure 8. Overview of architecture on the server side 


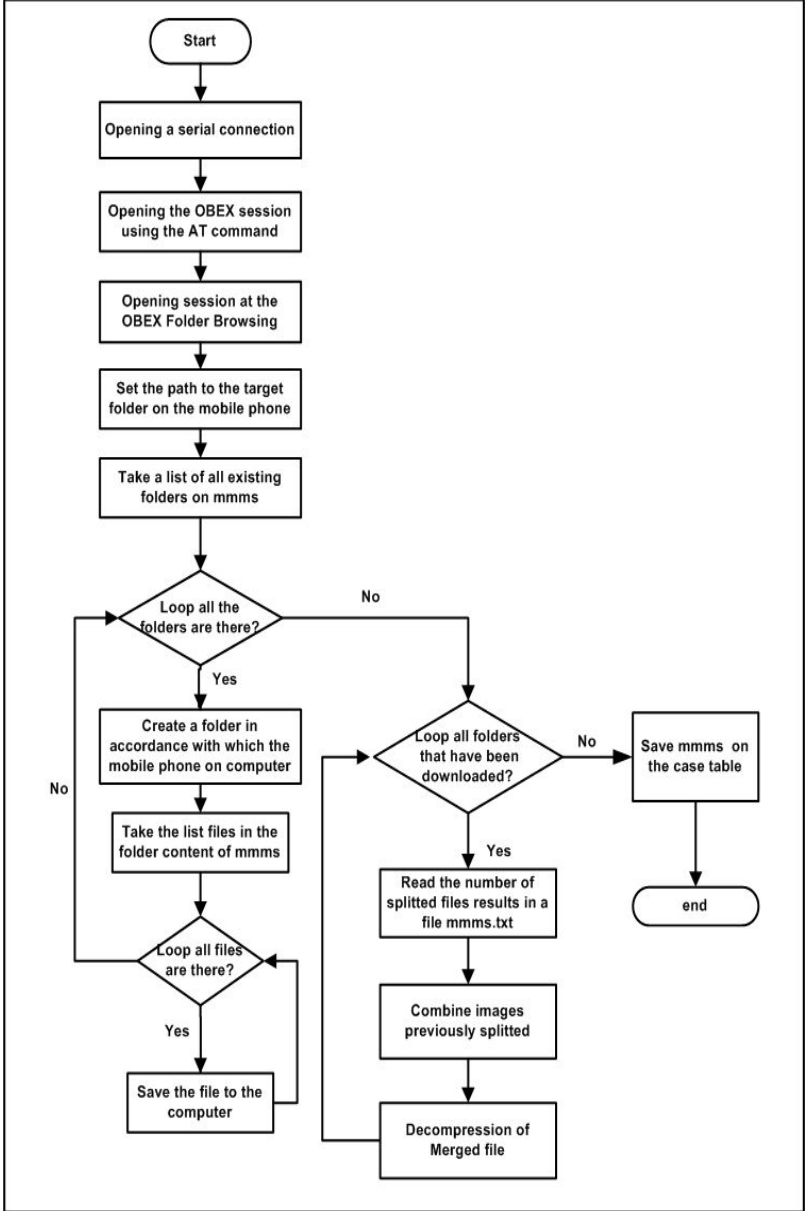

Figure 9. Improvement algorithm for downloading data

\section{IMPLEMENTATION AND SIMULATION}

In our previous paper [16], we have discussed about the implementation of compression and splitting techniques on the MMS application. In [17], we have discussed the implementation of MMS technology for streaming audio and video files. In this paper, we add cropping techniques for delivery of image files. This development aims to achieve an adaptive and cost-effective MMS framework for Internet applications. The cropping technique also ensures the good quality of the image which is critical for applications such as medical diagnosis. In general, the cropped part of the image may not be compressed as its size is normally small.

\section{A. Client Applications}

We use Java 2 micro edition (J2ME) programming language and Sony Ericsson mobile phone K630, J105i and J10i2 types to develop MMS application on the client side. This application can transmit multimedia files, such as images (JPEG, PNG, GIF and BMP), audio (AMR, WAV and AAC) and video (MP4, 3GP). Figure 10 shows MMS application for creating multimedia message.

We deliberately separate the MMS creation and sending applications in order to make the retransmission process of split files easier. The created messages are stored temporarily in the mobile phone till confirmation is received from the server. If confirmation is not received for any split-file, it will be retransmitted. The retransmitted splitfile will be merged with other split-files belonging to the

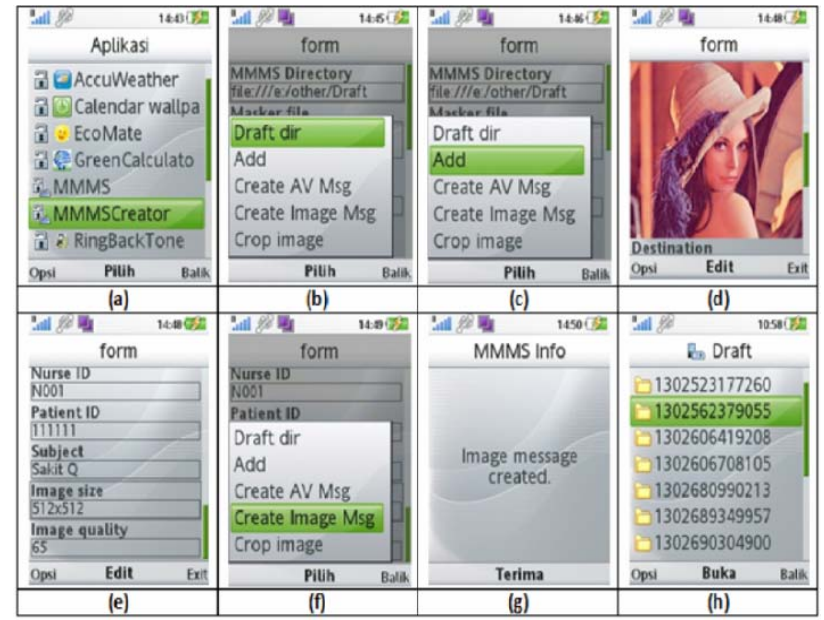

Figure 10. MMS application for creating multimedia message

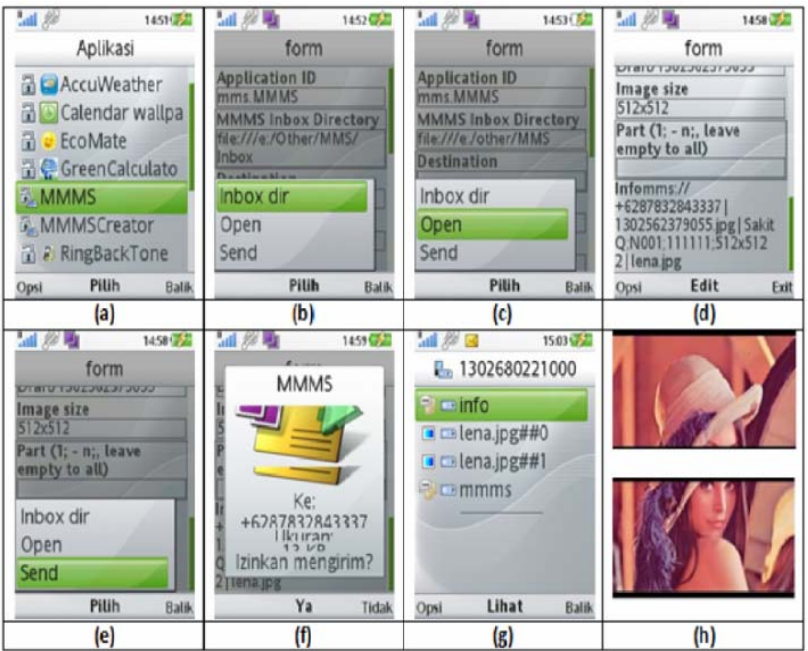

Figure 11. MMS application for sending multimedia message

same message identification. Sometimes, the sending process of split-files fails. This can happen due to disruption in network, disruption in MMS center or peak-traffic network of the operator used. This problem can be solved as follows: After server successfully downloads the whole message on the mobile phone gateway, it examines the successfully sent split files one by one using the information on the info.txt. If there any missing split files, the server will give a notification message using SMS technology to the sender and request for the missing files. Figure 11 shows the MMS application for sending multimedia messages.

We also develop cropping technique to answer the delivery address problems of the large image size. The cropping technique is used when only a small portion of the image area is needed. If we use resizing technique, it will certainly result in a significant loss of the quality. With the cropping technique, the image quality is preserved. The cropping technique results in a small file. This is almost the same as the one obtained with resize process but in this case the quality of image is preserved. This makes the developed system more adaptive and cost-effective. Figure 12 shows the implementation of cropping technique on the MMS application. 


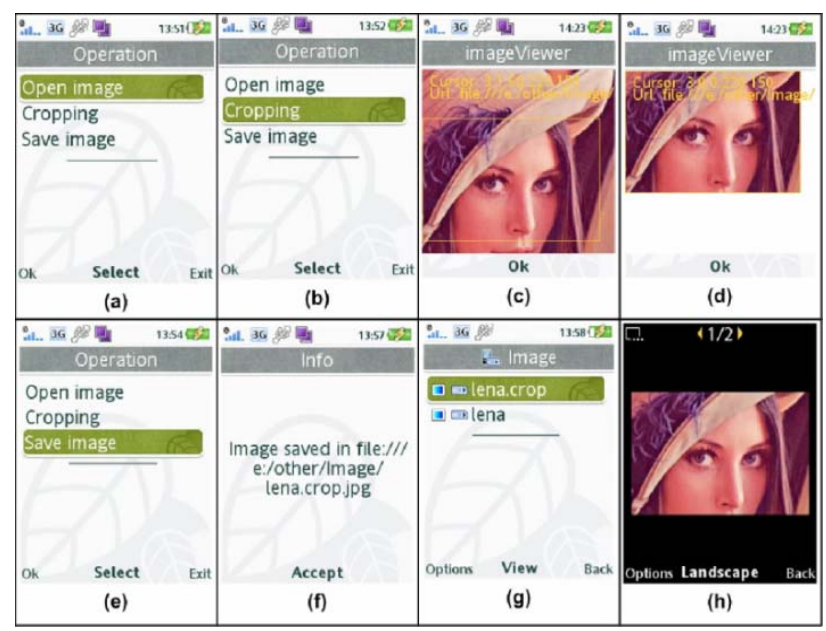

Figure 12. Implementation of cropping technique on the MMS

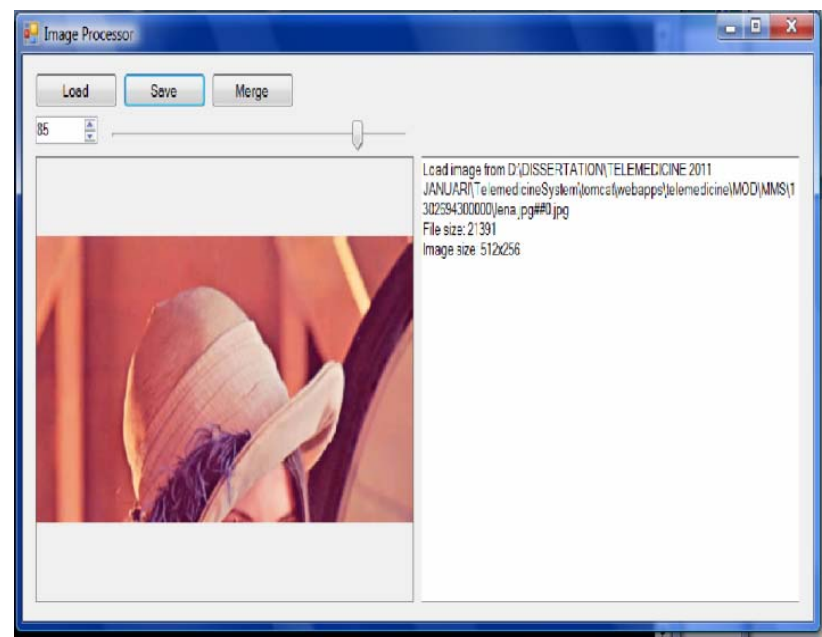

Figure 13. User interface for merging and decompression image

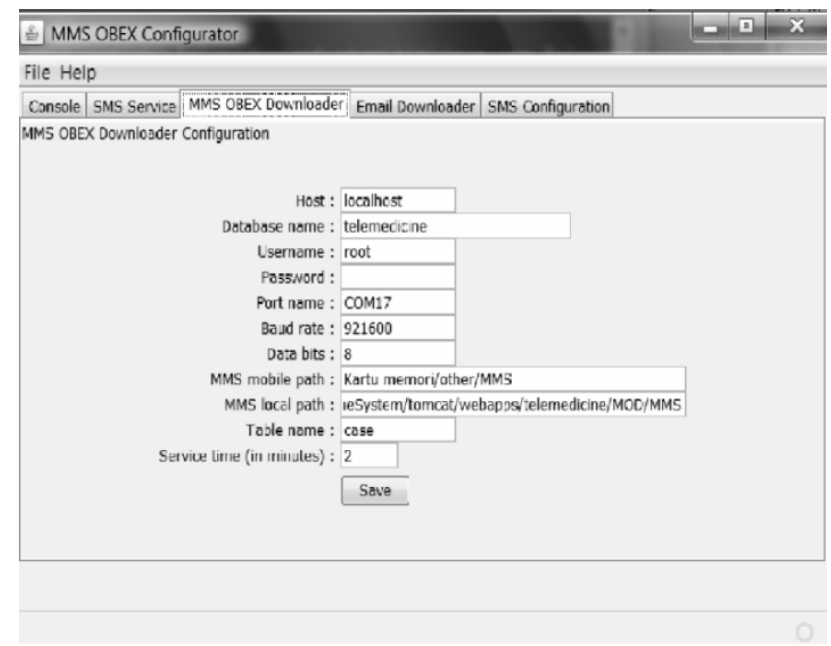

Figure 14. OBEX's User interface

\section{B. Server Applications}

On the client side, we use MMS application to send multimedia file to the server. For server application, we can develop any kind of Internet applications, such as telemedicine [18], e-commerce [19], e-learning [20], ebanking [21], etc. In this paper, we show how the merging and decompression processes occur on the server side.
Figure 13 shows the user interface for performing merging and decompression of JPEG image file to bitmap (BMP) image file.

We make a small program to perform merging and decompression processes. Our intention is to simulate and find out the results of each step of the merging and decompressing process before it is stored into the database server and to get more accurate data. This application is a small independent desktop application which performs merging and decompressing data processes successfully. In developing client-server application, the process of downloading, merging and decompressing is performed automatically by using OBEX that is installed on the server computer. Figure 14 shows the user interface for downloading, merging and decompressing data file on the server computer.

After the multimedia data is successfully stored into the database server, the data can be used to develop any kind of Internet application.

\section{EVALUATION AND ANALYSIS}

MMS technology will grow continuously in usage. Operators are facing challenges of taking appropriate measures to ensure that they keep pace with the rapid changes in order to optimize the users' experience and maximize the revenue benefit from multimedia messaging services. In the implementation of the development of a clientserver application, we use a network that has been provided by the operator. In developing MMS application is adjusted to the system need. It improves the ability of the MMS technology by applying several techniques such as compression, splitting, masking and cropping.

These techniques can be used not only for MMS technology but also for web-based services and technology such as the Hypertext Transfer Protocol (HTTP), File Transfer Protocol (FTP), Electronic Mail (EMail) or streaming. The bandwidth limitation on the internet or mobile network requires performing preprocessing on the mobile phone before the data is transmitted. We develop data transmission framework using MMS technology. It is because MMS technology can use general Packet Radio Service (GPRS) network which is used widely in rural and remote areas, while the 3G technology and latest mobile technology have not covered these areas yet. The GPRS coverage area is same like Global System for Mobile communications (GSM) mobile network, where it can cover almost all the areas in a cPHetaleveloped data transmission framework is not only to transfer larger file size through the network but also maintain the data quality. This research deals with implementation of techniques with an aim to achieve an adaptive and cost-effective method for transmitting data using MMS technology. The existing and the developed methods are then compared. The developed system is also adaptive to a variety of mobile internet applications.

We can use mobile phone device to access Internet applications easily, especially with the presence of smart phones that are flooding the market. Smart phones in general have wide display or screen therefore information can be seen more clearly than ordinary mobile phone. Web-based services use smart phones and provide facilities for mobile transactions. This method however requires large bandwidth and it needs the latest mobile technology such as Universal Mobile Telecommunications 
System (UMTS) or High-Speed Downlink Packet Access (HSDPA). If the mobile network available is only GPRS, Internet connection will be very slow. With GPRS, webbased services cannot be used in rural and remote areas that have limited bandwidth. The proposed framework is suitable for areas which have limited bandwidth and it also provides advantages with respect to issues such as security, integrity, privacy, portability and adaptability. In the developed framework, the use of smart phones and web-based services method are accommodated if sufficient bandwidth is available. The proposed method can be used on ordinary mobile phone if the availability of the bandwidth is limited.

We measure the quality of the data before and after transmission in order to determine whether the data quality is preserved or not. The data quality is influenced by the compression ratio that is used. The best data quality is obtained without compression. We use peak-signal to noise ratio (PSNR) and universal image quality index (UIQI) to measure the quality of the image. Tables II and III show the values of the quality measures after merging and after decompression respectively

The simulation results show that the proposed method can be successfully implemented to send large amount of data using MMS application without degrading the quality. It is proved by the PSNR values are more than $+30 \mathrm{~dB}$ and for splitting and cropping files before and after sending are identical.

The developed system is still a prototype system which can still be improved to adapt to the user needs. The main contribution of this research is to increase the capacity of files several times to transmit data from client to server. We have managed to simulate the developed application and installed in mobile phone device for using in multimedia message delivery. In this simulation, we use the maximum size $30 \mathrm{MB}$ using J2ME. This is a constraint of the use of Java technology that only has maximum size 30 KB. This means that the ability of sending MMS can be increased several-fold if more memory is available on the mobile phone. Table IV shows the comparison of the existing and developed frameworks.

We choose to develop client-server application using MMS technology because MMS has some advantages, such as (i) MMS needs lower bandwidth and lower sending cost compared to smart phones for accessing server applications, (ii) it needs ordinary mobile phones which are cheaper (iii) MMS data is more secured and also protected from unauthorized user compared to sending data through smart phone to the Internet application directly. We have explained the techniques in detail for transmitting large multimedia file using MMS technology. The modified techniques of the MMS technology can be used to develop practical client-server applications.

\section{CONCLUSION AND FUTURE WORK}

In this study, we have proposed a solution to solve the problem of size limitation in multimedia data transmission using MMS technology. The limitation of the maximum size restricts the use of the MMS technology severely. We cannot transmit good quality of data by using the existing technology.

In this paper, we have proposed a new framework for transmitting data using MMS technology for client-server application by implementing some techniques, such as compression, splitting, masking and cropping. The implementation of these techniques leads to an adaptive and cost-effective data transmission system. This method can be used to develop any kind of client-server application where the client is in mobile environment. For future work, we will develop and improve cropping technique that can be combined with compression and active contour model to produce automatic cropping for selected image object.

TABLE II.

PSNR AND UIQI BETWEEN COMPRESSION AND MERGING DATA

\begin{tabular}{|c|c|c|c|c|}
\hline \multirow{4}{*}{$\begin{array}{c}\text { Quality } \\
\text { Factor } \\
(\%)\end{array}$} & \multicolumn{2}{|c|}{ Lena.JPEG (512x512) size= 69214 Bytes } \\
\cline { 2 - 5 } & $\begin{array}{c}\text { On the } \\
\text { Client }\end{array}$ & $\begin{array}{c}\text { On the } \\
\text { Server }\end{array}$ & $\begin{array}{c}\text { On the Clien } \\
\text { OIQI }\end{array}$ & On the Server \\
\cline { 2 - 5 } & ffter Compression & After Merging & $\begin{array}{c}\text { After } \\
\text { Compression }\end{array}$ & After Merging \\
\hline 85 & 37.68 & 35.93 & 0.83 & 0.78 \\
\hline 80 & 37.07 & 35.41 & 0.8 & 0.75 \\
\hline 75 & 36.31 & 34.88 & 0.77 & 0.72 \\
\hline 70 & 35.88 & 34.31 & 0.75 & 0.7 \\
\hline 65 & 35.37 & 33.82 & 0.73 & 0.68 \\
\hline 60 & 35.02 & 33.71 & 0.71 & 0.66 \\
\hline 55 & 34.63 & 33.4 & 0.7 & 0.65 \\
\hline 50 & 34.37 & 33.09 & 0.69 & 0.64 \\
\hline
\end{tabular}

TABLE III.

PSNR AND UIQI BETWEEN COMPRESSION AND DECOMPRESSION DATA

\begin{tabular}{|c|c|c|c|c|}
\hline \multirow{4}{*}{$\begin{array}{c}\text { Quality } \\
\text { Factor }\end{array}$} & \multicolumn{3}{|c|}{ Lena.JPEG (512x512) size= 69214 Bytes } \\
\cline { 2 - 5 } $\mathbf{( \% )}$ & $\begin{array}{c}\text { On the } \\
\text { Client }\end{array}$ & On the Server & $\begin{array}{c}\text { On the } \\
\text { Client }\end{array}$ & On the Server \\
\cline { 2 - 5 } & $\begin{array}{c}\text { After } \\
\text { Compression }\end{array}$ & $\begin{array}{c}\text { After Merging \& } \\
\text { Decompression }\end{array}$ & $\begin{array}{c}\text { After } \\
\text { Compression }\end{array}$ & $\begin{array}{c}\text { After Merging \& } \\
\text { Decompression }\end{array}$ \\
\hline 85 & 37.68 & 43.60 & 0.83 & 0.93 \\
\hline 80 & 37.07 & 42.79 & 0.8 & 0.91 \\
\hline 75 & 36.31 & 42.17 & 0.77 & 0.90 \\
\hline 70 & 35.88 & 42.47 & 0.75 & 0.90 \\
\hline 65 & 35.37 & 42.27 & 0.73 & 0.89 \\
\hline 60 & 35.02 & 41.25 & 0.71 & 0.88 \\
\hline 55 & 34.63 & 41.49 & 0.70 & 0.88 \\
\hline 50 & 34.37 & 41.37 & 0.69 & 0.87 \\
\hline
\end{tabular}

TABLE IV.

COMPARISON OF THE EXISTING AND DEVELOPED FRAMEWORK

\begin{tabular}{|c|c|c|}
\hline Item & The Existing & The Developed \\
\hline Data Size & $30 \mathrm{~KB}$ & $>30 \mathrm{~KB}$ \\
\hline Language & Java 2 ME & Java 2 ME \\
\hline Quality & Reduced & Without reduced \\
\hline Technique & Resizing & Compression and Split \\
\hline Method & \begin{tabular}{|l}
$\begin{array}{l}\text { Single message every } \\
\text { sending }\end{array}$ \\
\end{tabular} & \begin{tabular}{|lll}
$\begin{array}{l}\text { Multiple message one } \\
\text { sending }\end{array}$ & \\
\end{tabular} \\
\hline Strengths & $\begin{array}{l}\text { Automatically } \quad \text { resizing } \\
\text { before sending }\end{array}$ & $\begin{array}{l}\text { - It can send data larger } \\
\text { than the existing } \\
\text { - Quality is not reduced }\end{array}$ \\
\hline Weakness & $\begin{array}{l}\text { - It cannot be modified } \\
\text { - It cannot send data } \\
\text { larger than } 30 \mathrm{~KB} \\
\text { - Quality and size are } \\
\text { reduced. }\end{array}$ & $\begin{array}{l}\text { - Risk of failure is still } \\
\text { high } \\
\text { - The big file is split to be } \\
\text { several file. }\end{array}$ \\
\hline
\end{tabular}




\section{REFERENCES}

[1] Article Snacth.com, http://www.articlesbase.com/technologyarticles/mms-the-best-messaging-technology-410230.html

[2] G. L. Bodic, Mobile Messaging Technologies and Services SMS, EMS, MMS, Second Edition, Jhon Wiley \& Sons, Ltd., 2005. http://dx.doi.org/10.1002/0470014520

[3] Comverse making your newtwork smarter, MMS is growing in More Ways than One: The Dimensions and Implicetions of Surging MMS Growth, pp. 1-9, 2009.

[4] G. L. Bodic, Multimedia Messaging Service: An Engineering Approach to MMS, Jhon Wiley \& Sons, Chichester, England 2003. http://dx.doi.org/10.1002/0470862521

[5] Open Mobile Alliance (OMA), Multimedia Messaging Service Requirement Candidate Version, OMA-MMS-ENC-V1_320080128-C, Approved Version 1.3, 2008.

[6] D. R. P. Graham, MMS Technology, Usage and Business Models, England, Jhon Wiley \& Sons Ltd., 2004.

[7] S. K. Samanta, J. Woods and M. Ghanbari, "Special Delivery: An Increase in MMS adoption”, IEEE Potentials, pp. 12-16, 2009. http://dx.doi.org/10.1109/MPOT.2008.930442

[8] A. Setyono, M. J. Alam and R. A. Al-Saqour, Evaluation and Analysis of Multimedia Messaging Service (MMS) Framework in Message Delivery, CITA’09, pp. 241-245, 2009.

[9] K. James, and W. Keith, Computer Networking, a Top-Down Approach Featuring the Internet, Addison Wesley, 2010.

[10] C. Salazar and T. D. Tran, “A Complexity Scalable Universal DCT Domain Image Resizing Algorithm”, IEEE Transactions On Circuits And Systems For Video Technology, Vol. 17, No. 4, 2007. http://dx.doi.org/10.1109/TCSVT.2007.893836

[11] C. Saravanan, Color Image to Grayscale Image Conversion, Second International Conference on Computer Engineering and Applications, pp. 196-199, 2010. http://dx.doi.org/10.1109/ICC EA.2010.192

[12] F. Ebrahimi, M. Chamik and S. Winker: JPEG vs. JPEG2000: An Objective Comparison of Image Encoding Quality, Genista Corporation, 1-9, 2005.

[13] A. Setyono, M. J. Alam and R. A. Al-Saqour, Split and Merge for Multimedia Messaging Service, The $2^{\text {nd }}$ International Conference on Engineering and ICT, 2010.

[14] I. F. Jafar and K. A. Darabkh, "A Modified Unsharp-Masking Technique for Image Contrast Enhancement”, IEEE, 2011.

[15] S. Bongwon, L. Haibin, B. Benjamin, and W. David, "Automatic Thumbnail Cropping and its Effectiveness”, ACM Letters Chi Vol. 5 Issue 2, 2003.
[16] A. Setyono, M. J. Alam and C. Eswaran, Development and Implementation of Compression and Split Techniques for Multimedia Messaging Service Applications, International Conference on Intelligent Network and Computing, 2010.

[17] A. Setyono, M. J. Alam and C. Eswaran, Development of Streaming Application for Mobile Telemedicine System Using Multimedia Messaging Service Technology, International Conference on Computer and Communication Devices, 2011.

[18] A. A. Tahat, Mobile Personal Electrocardiogram Monitoring System and Transmission Using MMS, Proceeding International Caribbean Conference on Device, 2008.

[19] C. Lei-da, and S. Gordon, Mobile Commerce Development Application. Cybertech Publishing. USA, 2005.

[20] M. H. Hasan, N. S. Haron, N. Syafiqoh, and S. M. Yazid: Development of MMS-Based Examination Results System, Proceeding of the $9^{\text {th }}$ WSEAS International Conference on Applications of Computer Engineering, pp. 157-163, 2010.

[21] M. H. Hasan and A. Khalid., "Development of MMS-Based Receipt System for Mobile Banking”. IEEE, 2010.

\section{AUTHORS}

A. Setyono is a Ph.D student by research from Faculty of Information technology at Multimedia University, Malaysia. He is also a lecturer of Faculty of Computer Science at Dian Nuswantoro University, Indonesia. (email: setyonoandik@ gmail.com).

M. J. Alam is a lecturer from Faculty of Information Technology at Multimedia University, Malaysia. His research interests are human computer interaction, image processing, medical image technology, 3D reconstruction, face recognition and gait recognition. (e-mail: md.jahangir.alam@mmu.edu.my).

C. Eswaran is a professor of Faculty of Information Technology and chairman of Center for Multimedia Computing at Multimedia University, Malaysia. He served as professor in the department of electrical engineering at Indian Institute of Technology, Madras, India. (e-mail: eswaran@mmu.edu.my).

Received 23 July 2011. Published as resubmitted by the authors 14 November 2011. 